\title{
High resolution solar magnetometry with the spectrograph of the Pic du Midi Turret Dome
}

\author{
J.-M. Malherbe ${ }^{1}$, Th. Roudier², P. Mein ${ }^{1}$, J. Moity ${ }^{1}$, and R. Muller ${ }^{2}$ \\ 1 LESIA, Observatoire de Paris, Section de Meudon, 92195 Meudon Cedex, France \\ e-mail: Jean-Marie.Malherbe@obspm.Fr \\ ${ }^{2}$ Laboratoire d'Astrophysique, Observatoire Midi Pyrénées, 57 Avenue d'Azereix, PB 826, 65008 Tarbes Cedex, France
}

Submitted 6 May 2004 / Accepted 23 July 2004

\begin{abstract}
We present the first results obtained with a new Ferroelectric Liquid Crystal (FLC) polarimeter operating with the spectrograph of the Pic du Midi Turret Dome, since September 2003. We observed the solar granulation around active region NOAA 0459 with the Multichannel Subtractive Double Pass (MSDP) operating mode and analysed the circular polarization of the Na D1 $589.6 \mathrm{~nm}$ spectral line in terms of longitudinal magnetic fields in a 2D field of view $(16 \times 142 \mathrm{arcsec})$. Image quality was fairly good and limited to 0.4 arcsec due to the pixel sampling. This observation reveals the presence of magnetic concentrations of several hundred Gauss which are mainly located in the intergranular lanes. Data analysis performed at two different optical depths (line core and line wings) also suggests that magnetic lines are curved and diverge with increasing altitude.
\end{abstract}

Key words. Sun: photosphere - Sun: granulation - Sun: magnetic fields - instrumentation: polarimeters

\section{Introduction}

Most of the magnetic flux crossing the photosphere in the quiet Sun is concentrated in small elements (i.e. flux tubes) which are at the limit of resolution of the present ground-based solar telescopes. Up to now, in order to benefit from the best spatial resolution on magnetic structures, visible-light signatures or "proxies" of the magnetic field have been extensively used. These proxies refer to small bright features observed as field patches or as small bright points (Muller \& Roudier 1984; Van Ballegooijen et al. 1998; Berger \& Title 2001; Nisenson et al. 2003). The magnetic flux tubes are dynamic features, and their inclination relative to the line of sight varies in time (Steiner et al. 1998). In consequence, brightness fluctuations up to the extinction of the proxy may occur and can be explained by geometrical effects which do not allow us to observe continually the proxy in intensity.

Thus, the study of the evolution of magnetic elements and their interaction with convection requires magnetograms with high spatial resolution and fair sensitivity (better than 20 Gauss or 0.002 Tesla). Some previous works in the measurement of the magnetic field at high spatial resolution have been performed by different authors. Simultaneous direct observation of white light proxies and magnetic fields allowed them to establish the link between these two quantities for the solar flux tubes (Muller et al. 2000; Keller 1992). Zhang et al. (1998) found that most of the bright features such as filigree in photospheric filtergrams are related to corresponding magnetic features. These magnetic concentrations show fast changes within several minutes. Stolpe \& Kneer (2000) found weak fluxes almost everywhere in the granular pattern with a preference $(65 \%)$ for the intergranular spaces. The speckle observations performed by Koschinsky et al. (2001) of the smallscale magnetic structures revealed that, in the quiet sun, the granular dynamics dominates the time evolution of the magnetic elements. The magnetic flux emerges on the smallest resolvable scale length (less than $1 \mathrm{Mm}$ ), and its dispersion by granular motions was confirmed by De Pontieu (2002). Very recently, Dominguez Cerdeña et al. (2003) confirmed using speckle restoration and high spatial resolution magnetograms ( $0.5 \mathrm{arcsec})$ that $45 \%$ of the observed area is covered by patches with a magnetic field above the noise. More precisely they suspected the presence of structures with field strengths larger than 1000 Gauss, which could occupy only $2 \%$ of the surface. The observations (Dominguez Cerdeña et al. 2003) and the numerical simulations (Cattaneo 1999; Emonet \& Cattaneo 2001; Schrijver \& Zwann 2000) pointed out that most of the magnetic field could be generated by local dynamo. This results shows the great interest is learning, at high spatial resolution, the properties and the dynamics of the magnetic field and its interaction with the solar turbulent convection.

We present in this paper then new capabilities of high resolution solar magnetometry obtained with the spectrograph of the Pic du Midi Turret Dome, which allows us to get magnetograms and dopplergrams approaching the limit of the resolution of the refractor. 


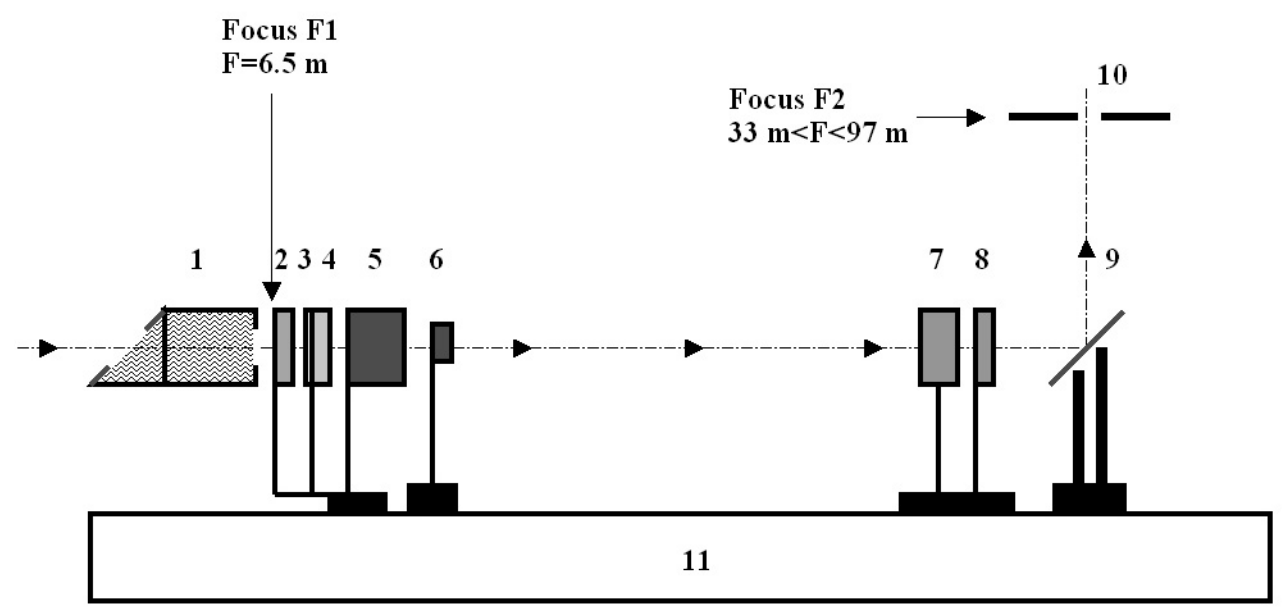

1: water cooling device (field stop)

2: static quarter wave plate

3: UV/IR filter $(390-700 \mathrm{~nm})$

4: half wave FLC modulator

5: magnification lens $(f=40 / 60 / 95 \mathrm{~mm})$

6: beam shifter and flat field lenses (MSD)
7: interference filter

8: precision dichroïc linear polarizer

9: flat mirror

10: spectrograph entrance slit/window

11: optical rail

$P$ only)

Fig. 1. The optical beam between the primary focus F1 and the secondary focus F2 (the $50 \mathrm{~cm}$ refractor is located on the optical axis at left).

\section{Experimental setup}

The Pic du Midi Turret Dome is a $50 \mathrm{~cm}$ aperture refractor (focal length of $6.45 \mathrm{~m}$ at the primary focus F1) supported by an equatorial mount. The beam has a cylindrical symmetry along the optical axis. Polarization analysis is achieved before transmission to the spectrograph by a flat mirror (Fig. 1). Therefore instrumental polarization is minimized. The resolving power (including the refractor and the spectrograph) is 0.3 arcsec in the yellow part of the spectrum. The primary image is magnified between 5 to 15 times at the secondary focus F2 where the entrance window of the spectrograph is located, according to the focus of the magnification lens. We used a new prototype of liquid crystal polarimeter between focus F1 and F2. It was used for the first time in September 2003. The polarimeter receives white light and has the following elements (Fig. 1):

- A half wave achromatic polarization rotator (400 to $700 \mathrm{~nm}$ ) made by Displaytech (USA) which combines a single Ferroelectric Liquid Crystal (FLC) cell with a static birefringent element; it takes less than one millisecond for the controller to switch between zero to half wave using fast transient nematic effect $( \pm 15 \mathrm{~V}$ peak to peak) within low voltage DC modulation $( \pm 5 \mathrm{~V})$. The FLC is protected by a pass band filter in order to avoid damage of the crystal by the Ultra Violet radiation (below $380 \mathrm{~nm}$ ), and heating by infrared radiation (above $700 \mathrm{~nm}$ ). The temperature of the FLC is, at the moment, not yet controlled.

- A static and achromatic zero order quarter wave plate (400 to $700 \mathrm{~nm}$ ) from Fichou (France). The waveplate, at the wavelength of observation $(589.6 \mathrm{~nm})$, is of course not exactly quarter wave, but the departure is very small and less than $0.7 \%$, according to the calibration.

- A dichroïc linear polarizer from Fichou (France), located at the exit of the system, on the optical axis, before the reflecting mirror to the spectrograph. The acceptance axis is orthogonal to the long direction of the entrance window in order to be orthogonal to the rules of the Echelle grating of the spectrograph (for maximum luminosity). The precision of the polarizer is of the order of $0.05 \%$ (this value represents light transmission through two crossed polarizers).

The static quarter wave plate, in combination with the FLC, allows modulation from quarter to three quarter waves, providing analysis of the circular polarization of light by the linear polarizer. Hence, Stokes parameters $I+V$ and $I-V$ are obtained sequentially from the output signal $S=\frac{1}{2}(I-Q \sin \delta+V \cos \delta)$ where $\delta=(0, \pi)$ is the retardance of the FLC. When the quarter wave plate is not mounted, the polarimeter allows modulation from zero to half wave, for linear polarization analysis. $I+Q$ and $I-Q$ are obtained from the output signal $S=\frac{1}{2}(I+Q \cos \delta-V \sin \delta)$, with $\delta=(0, \pi)$. This feature is frequently used for observations of the second solar spectrum at the limb, which will be presented in another paper.

We use the $8 \mathrm{~m}$ mirror spectrograph in MSDP mode as described by Mein $(1981,1991,2002)$. In this system, the thin slit usually used in spectrographs is replaced by a $2 \mathrm{D}$ entrance aperture, covering in our case a solar area of $16 \times 142$ arcsec with the intermediate magnification lens that we used (equivalent focal length of $65 \mathrm{~m}$ at focus F2). The dispersive element is a grating ( 300 rules $/ \mathrm{mm}$, blaze angle $63^{\circ} 26^{\prime}$ ) working at order 10 for Sodium lines. The order is isolated by an interference filter (10 nm bandwidth). The light is dispersed two times by 
the grating. At the first pass (the dispersion is about $4.2 \mathrm{~mm} / \AA$ ), a multi slit beam shifter device selects and shifts simultaneously $112 \mathrm{D}$ channels (the distance between channels is equal to $0.6 \mathrm{~mm}$, this constant governs the spectral resolution of observations, i.e. $144 \mathrm{~m} \AA$ here). At the second pass, dispersion is subtracted so that 11 non monochromatic images of the solar area form at the focus of the spectrograph (see Roudier et al. 2003, and Fig. 3), in 11 spectral ranges separated by $144 \mathrm{~m} \AA$.

The MSDP image obtained at the focus of the spectrograph is then reduced to form on a CCD camera from LaVision (Germany) with temperature control (Peltier cooling at $-10^{\circ} \mathrm{C}$ ). The pixel size on the CCD was 0.2 arcsec in our setup. Each pixel can accumulate up to 25000 electrons corresponding to a dynamic of 12 bits (readout noise of 7 electrons). In general, we work in the continuum at 0.75 times the saturation level giving approximately a signal to noise ratio slightly better than 100 or a photometric accuracy better than $10^{-2}$. The exposure time is the shortest as possible in order to freeze the turbulence and was $30 \mathrm{~ms}$ during our run.

Our polarimetric observations consisted of shooting sequentially as fast as possible pairs of MSDP images $I+V, I-V$. The modulator, in theory, is able to switch between two states of polarization at a frequency of a few $\mathrm{KHz}$. However, we are limited by the speed of our CCD camera and data acquisition system, which allowed us to work at the maximum speed of 4 images/s, including modulation. In order to have the possibility of image selection and to improve the magnetic sensitivity, we make, in practice, on the same field of view bursts of couples $I+V, I-V$, as fast as possible. In September 2003, we recorded bursts of 10 couples (as we were limited by memory) and we present in this paper the analysis we made after image selection (best image quality), and after data accumulation and destretching (best magnetic sensitivity).

The flat field is difficult at the Turret Dome because the spectrograph is attached to the refractor and changes position with time, producing small mechanical shifts. That is the reason why we do not practice this as with telescopes with non moving spectrographs. We automatically incorporate for the flat field in the beam, at the pupil location (Fig. 1), a defocusing lens together with a prism in order to shift the field of view by about 1 or 2 arcmin alternatively north and south of the observed area. This process has no influence on the pupil and is made systematically for each set of observations. The two flat images are averaged in the data processing and are of course taken in the two states of polarization.

In order to retrieve with a good accuracy (better than half a pixel) the borders of the channels, corresponding to the entrance window of the spectrograph, we also take a field stop image for each set of observations. This is done by automatically replacing the interference filter by a neutral density filter, in order to remove the observed spectral line from the field of view.

Hence, an elementary set of data consists of a burst of couples $I+V, I-V$ of the same region of interest, plus two flat field images and a field stop image obtained to the north and south, also in the two states of polarization. A complete sequence is a series of elementary sets of data obtained periodically, and terminated by a dark current image.

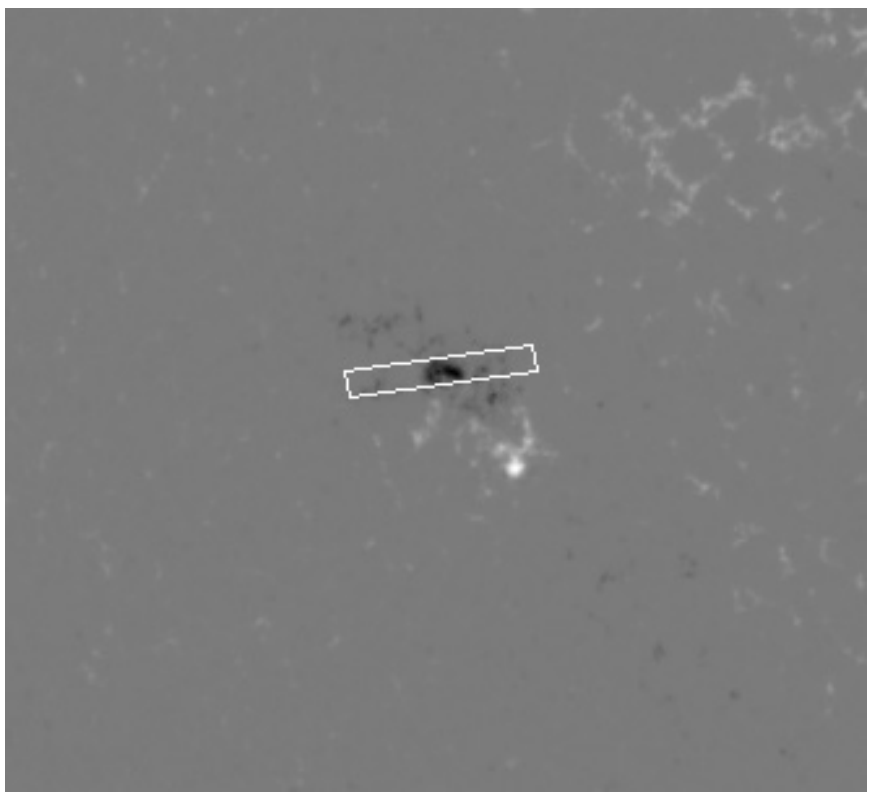

Fig. 2. SOHO/MDI magnetogram on 2003 September 19 at 11:17 UT, showing NOAA 0459 (part of the full disk magnetogram, size of $11 \times$ 10 arcmin, North is up, West is left, pixel size of 2 arcsec). We observed with the MSDP the northern spot (the white box corresponds to the MSDP field of view); the magnetic field found by MDI in this spot was between -600 and -1000 Gauss, in agreement with our results (courtesy SOHO/MDI).

\section{Observations and data reduction}

We observed active region NOAA 0459 (West 20, South 10) on 2003 September 19, with fairly good seeing conditions. We made several series of bursts and selected the best one. We have a set of 10 couples $I+V, I-V$, flat field, field stop and dark current. There is a sunspot in the middle of the field of view $(16 \times 142 \operatorname{arcsec})$. The observed region is unfortunately not located in the $8 \times 8$ arcmin square area of SOHO/MDI but was visible only on full disk magnetograms (Fig. 2). It appears as a bipolar region, but we had only the northern part in the field of the MSDP (Fig. 3), representing only one polarity (the negative one). The entrance window has a direction parallel to the geographic East West direction, and makes a small angle with the solar East West direction ( $P$ angle of $24.5 \mathrm{deg}$ ). The sampling pixel was 0.2 arcsec on the CCD detector with $30 \mathrm{~ms}$ exposure time. A GIF movie, showing the 20 images of the burst with alternate polarizations, that we analyse here, is available at http://helios.obspm.fr/malherbe/AA20030919/ msdp.gif, and clearly shows the Zeeman effect on the sunspot as an oscillation of the spectral line.

Data analysis was performed using the standard MSDP software developed by P. Mein and available on line at the BASS2000 national data base (see http://bass2000. bagn . obs-mip. fr). The following steps are made for each state of polarization:

\section{- field stop images:}

Such images do not contain any spectral lines (as the interference filter is removed) and are used for the precise detection of the borders of the 11 channels, for subsequent 


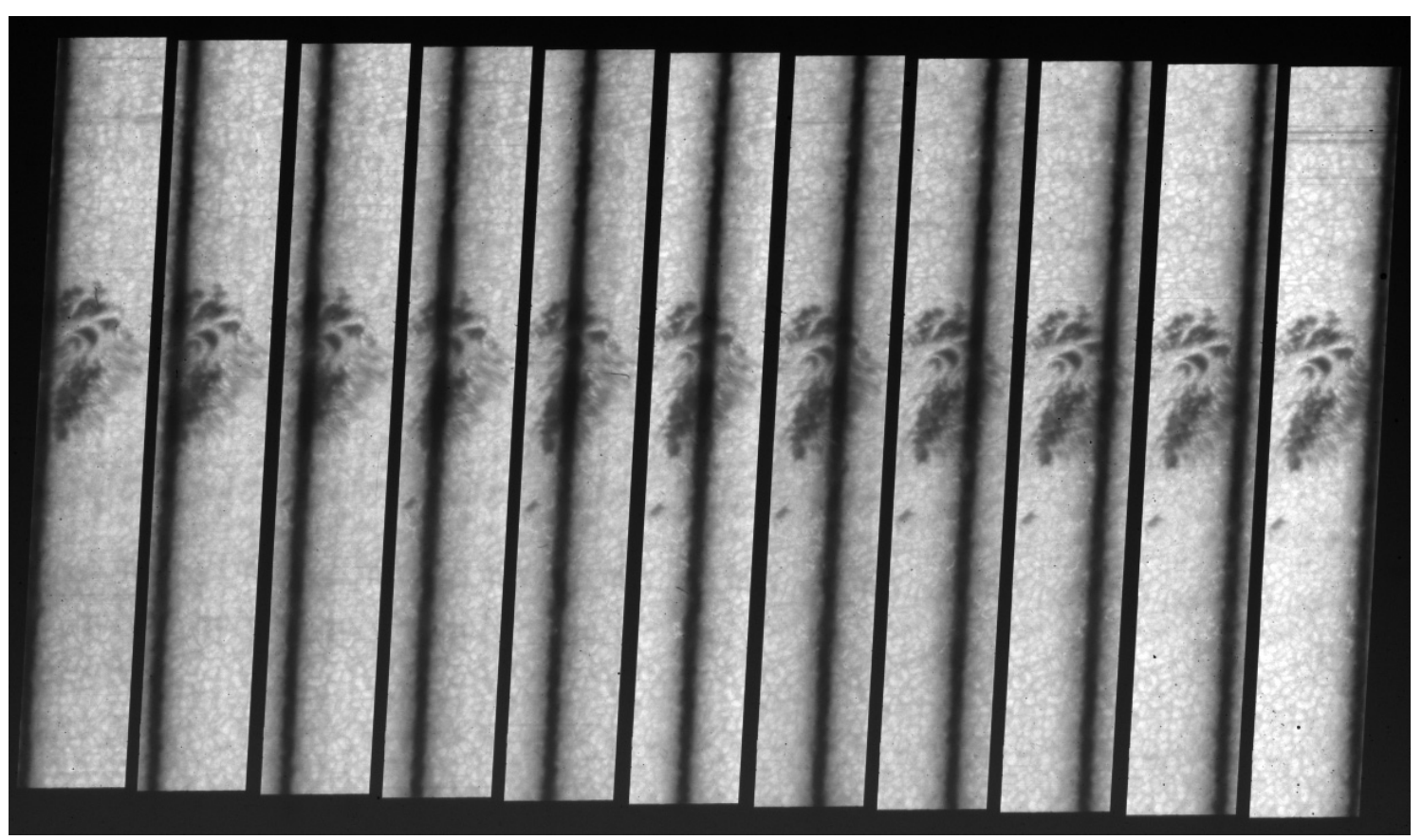

Fig. 3. MSDP image obtained on 2003 September 19 at 09:07 UT, showing the northern part of NOAA 0459 and the 11 simultaneous channels with the NaD1 $589.6 \mathrm{~nm}$ spectral line. The global wavelength shift between each channel is $144 \mathrm{~mA}$. The channels are not monochromatic, and the wavelength increases, inside each channel, from the left to the right. The pixel size is 0.2 arcsec and the field of view $16 \times 142$ arcsec. North is right. (better quality image available at http://helios.obspm. fr/malherbe/AA20030919/msdp.jpg).

superposition. The precision is better than 0.5 pixel $(0.1 \mathrm{arcsec})$. The geometry determined at this stage is applied to flat field images and to observations.

- flat field images:

Flat field images are performed using defocusing lenses in the pupil. They provide, for each pixel of the solar area, a reference profile of the NaD1 line. Flat field images are also used to determine the curvature of the spectrum, and some optical defaults such as the presence of transversalium or transmission differences between the 11 channels.

- observations:

The line profile (with 11 points separated by $144 \mathrm{~m} \AA$ ) is determined for each point of the field of view, and compared to the corresponding reference profile derived from the flat field. Using the bisector method based on searching the points of equal intensity in the profile, separated by $2 \Delta \lambda$, we can define the intensity fluctuation in $\mathrm{NaD} 1 \pm \Delta \lambda$ in comparison to intensity found with the same band in the reference profile. Hence, we are able to compute several maps of intensity fluctuations: line centre, $\pm 144 \mathrm{~m} \AA$ (below inflexion points), and $\pm 288 \mathrm{~m} \AA$ (above inflexion points). The middle of the band defines the position of the line in wavelength; the displacement between the current and reference profile provides the line shift in NaD1 $\pm \Delta \lambda$. As for intensity fluctuations, we obtain maps of line shifts at $\pm 144 \mathrm{~m} \AA$ (below inflexion points) and $\pm 288 \mathrm{~m} \AA$ (above inflexion points). Thus, images of the continuum were produced using the bluest channel of the MSDP (channel 11), but, when considering a single channel, one gets a nonmonochromatic picture in which the wavelength varies along the small dimension (direction of dispersion). Line displacements can be interpreted in terms of Dopplershifts (velocities) or in terms of Zeeman splitting (magnetic field).

According to this method, we compute maps of line shifts both from $I+V$ profiles and $I-V$ profiles. The half sum of displacements $\left(\Delta \lambda_{v}=\left(\Delta \lambda_{+}+\Delta \lambda_{-}\right) / 2\right)$ derived from $I+V$ and $I-V$ gives the line of sight velocity $V / /$ (according to the usual formula $\left.V / /=C \Delta \lambda_{v} / \lambda\right)$, and the half difference between $I+V$ and $I-V$ shifts $\left(\Delta \lambda_{B}=\left(\Delta \lambda_{+}-\Delta \lambda_{-}\right) / 2\right)$ gives the line of sight magnetic field, under the assumption that the two maps can be exactly superimposed. As the delay between $I+V$ and $I-V$ is of the order of $0.2 \mathrm{~s}$, this is only true in first approximation, because of turbulence and seeing effects. Velocities and magnetic fields computed with this method are called in the following as raw results. The magnetic field was computed by the classical formula giving the Zeeman splitting of the $\sigma$ components, in wavelength units:

$\Delta \lambda_{B}=4.6710^{-13} \Delta g B \lambda^{2}$

with $B$ in Gauss, $\lambda$ and $\Delta \lambda$ in $\AA$. $\Delta g=1.33$ is the Landé factor of the NaD1 transition. This formula numerically gives an equivalence between the displacements due to Doppler or Zeeman effects: $1 \mathrm{~m} / \mathrm{s} \approx 0.91$ Gauss.

A second approach consists of determining the spatial shift between continuum images of $I+V$ and $I-V$ couples, because the solar granulation is clearly visible; the precision is a fraction of a pixel, using cross correlation. We applied the shift measured between the continuum images to the spectral line displacements before velocity and magnetic field calculation.

But a much better result, in terms of signal to noise ratio, is obtained with both correlation and destretching between 


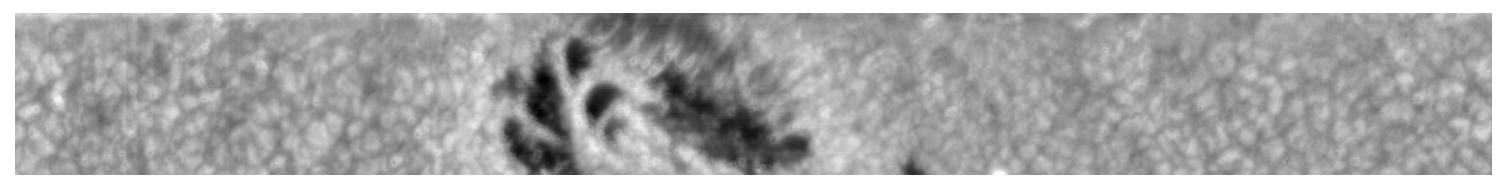

Fig. 4. MSDP image in the blue continuum of the NaD1 line, corresponding to channel 11 (this means that this image is not strictly monochromatic). The pixel size is $0.15 \mathrm{arcsec}$ and the field of view is $16 \times 142 \mathrm{arcsec}$. North is up.
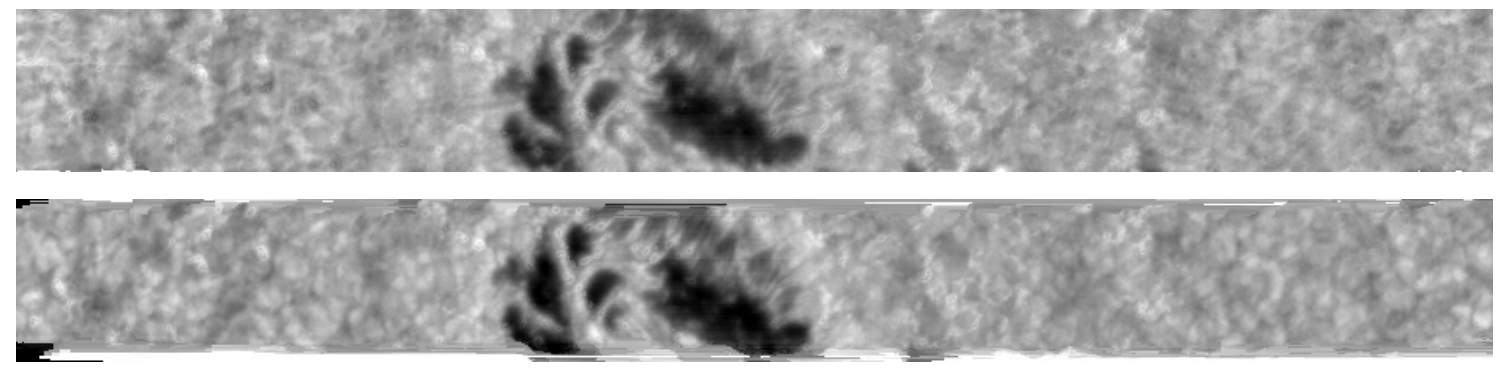

Fig. 5. Intensities in $\mathrm{NaD} 1 \pm 144 \mathrm{~m} \AA$ and $\pm 288 \mathrm{~m} \AA$ (in this latter case, the width of the field of view is slightly reduced due to instrumental limitations in the wings).

continuum images, and then applying the results to line displacement maps; we used for that purpose the code developed by November (1986) and modified by Vigneau (private communication). This method has also a great advantage: it is possible to use all images of a burst to increase the magnetic sensitivity by summing data after destretching.

\section{Results}

Although the sampling pixel size is $0.2 \operatorname{arcsec}(145 \mathrm{~km})$, we used in the data processing an output pixel of only 0.15 arcsec $(110 \mathrm{~km})$ in order to limit the loss of quality due to numerous geometrical and spectral interpolations done by the software. Figure 4 shows the blue continuum of the field of view obtained from channel 11, and exhibits clearly the sunspot and the granular pattern around it. We used this image as the reference image of the bursts of 10 couples $I+V, I-V$, and in the following, cross correlation and destretching between continuum images were performed using this reference frame.

We show in Fig. 5 the intensity obtained in NaD1 $\pm 144 \mathrm{~m} \AA$ (the granulation disappears, as expected, near the line core) and $\pm 288 \mathrm{~m} \AA$ (close to the continuum height). According to Roudier et al. (2001), the difference of altitude between these two positions in the spectral line is of the order of $160 \mathrm{~km}$, a value obtained from modelling the radiative transfer of $\mathrm{NaD} 1$ through the VAL-C atmospheric stratification with no magnetic field. This first approximation value is of the order of 1.5 pixel $(0.22 \mathrm{arcsec})$. We do not see, contrarily to Roudier et al. (2003), numerous bright points (filigree) at $\pm 144 \mathrm{~m} \AA$, probably because they had a better spatial resolution and sampling (0.13 arcsec instead of $0.20 \mathrm{arcsec}$ ). Indeed, Topka et al. (1992), and Title \& Berger (1996) studied bright point contrasts in continuum wavelengths and suggested that flux tubes could be smaller than 0.15 arcsec; the resolution of our observations may be too low to distinguish with good contrast flux tubes in intensity, but sufficient to see their signature in terms of magnetic flux.
Figure 6 shows the raw radial velocities obtained in $\mathrm{NaD} 1 \pm$ $144 \mathrm{~m} \AA$ and $\pm 288 \mathrm{~m} \AA$, resulting from the sum of line shifts derived from sequential $I+V$ and $I-V$ observations. Velocities exhibit the classical 5 min oscillation pattern (which we were not able to filter, because we do not have a temporal sequence); the solar granulation is no longer visible with increasing height (as at $\pm 144 \mathrm{m \AA}$ ).

Figures 7 and 8 show the line of sight magnetic field obtained in $\mathrm{NaD} 1 \pm 144 \mathrm{~m} \AA$ and $\pm 288 \mathrm{~m} \AA$, resulting from different methods which are described in detail in the following:

- the difference of line shifts derived from two sequential $I+V$ and $I-V$ observations which are strictly superimposed (Figs. 7 and 8, top). We estimated the noise around 50 Gauss at $\pm 144 \mathrm{~m} \AA$ and 130 Gauss at $\pm 288 \mathrm{~m} \AA$. In the sunspot, we found results in agreement with MDI full disk data at $\pm 288 \mathrm{~m} \AA$, and about 2 times smaller at $\pm 144 \mathrm{~m} \AA$ (higher altitude); but our spatial resolution is 10 times better, making comparison difficult. We now focus on the small scale magnetic concentrations which appear outside the sunspot. At $\pm 144 \mathrm{~m} \AA$, the line of sight magnetic field $B / /$ (measured inside 47 magnetic elements smaller than $0.5 \mathrm{arcsec}$ ) is in the range -650 Gauss to -310 Gauss, with a mean value of -480 Gauss; the corresponding radial velocities $V / /$ are more dispersed, from $-1250 \mathrm{~m} / \mathrm{s}$ to $+500 \mathrm{~m} / \mathrm{s}$, with a mean value of $-220 \mathrm{~m} / \mathrm{s}$ (downflows). At $\pm 288 \mathrm{~m} \AA, B / /$ is in the range -1580 Gauss to -570 Gauss, with a mean value of -945 Gauss; the corresponding velocities $V / /$ are in the range $-2400 \mathrm{~m} / \mathrm{s}$ to $+730 \mathrm{~m} / \mathrm{s}$, with a mean value of $-500 \mathrm{~m} / \mathrm{s}$ (downflows). We noticed that the magnetic concentrations at $\pm 144 \mathrm{~m} \AA$ do not exactly superimpose on magnetic elements at $\pm 288 \mathrm{~m} \AA$, suggesting, in agreement with Topka et al. (1992) that field lines could be significantly inclined or curved. We generally have a shift of one or two pixels, representing a distance of 100 to $200 \mathrm{~km}$, which can be related, in order of magnitude, to the difference of altitude $(160 \mathrm{~km})$ between the two levels. We also 

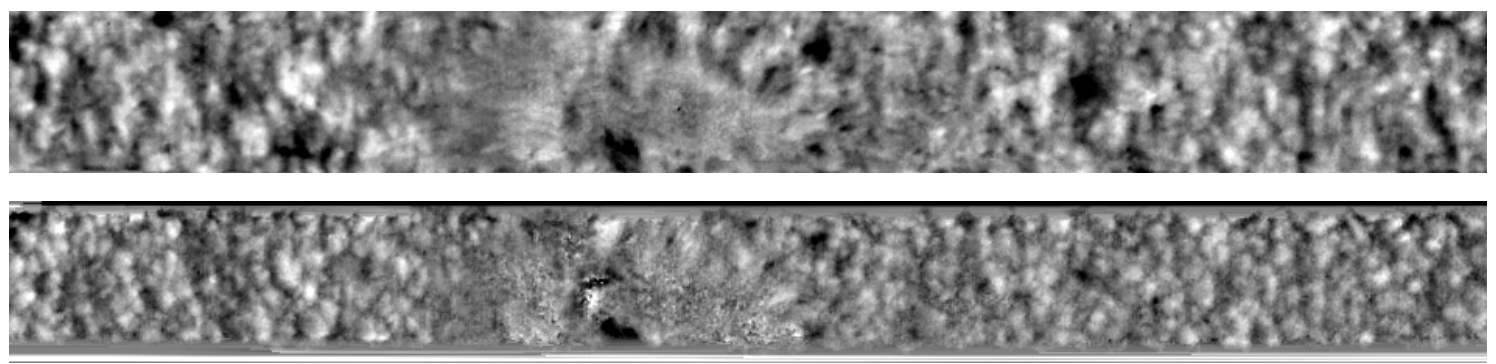

Fig. 6. Radial velocities in $\mathrm{NaD} 1 \pm 144 \mathrm{~m} \AA$ and $\pm 288 \mathrm{~m} \AA$ (in this latter case, the width of the field of view is slightly reduced due to instrumental limitations in the wings). Upflows are white and downflows are dark.
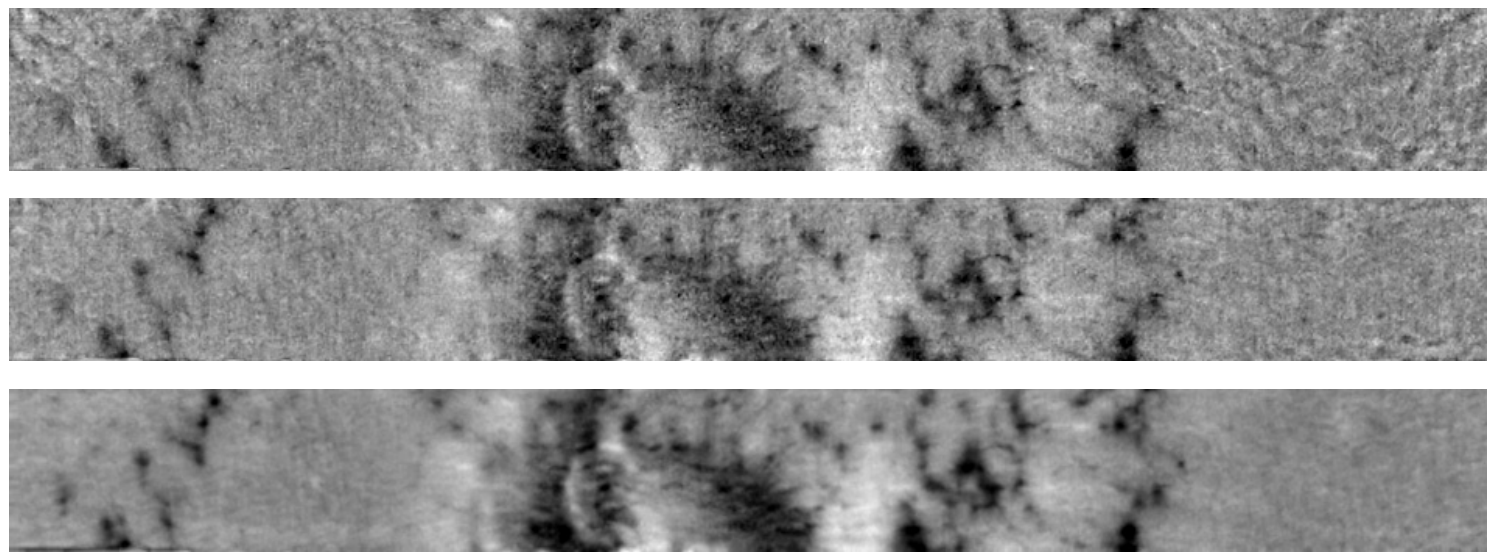

Fig. 7. Line of sight magnetic field $B / /$ in $\mathrm{NaD} 1 \pm 144 \mathrm{~m} \AA$. North polarities are white and south polarities are dark. Top: $B / /$ is derived directly from the difference of line shifts measured on $I+V$ and $I-V$; middle: $B / /$ is obtained by the same method, but after cross correlation and destretching; bottom: $B / /$ is computed from a burst of 10 couples $I+V, I-V$, after cross correlation and destretching, increasing sensitivity.
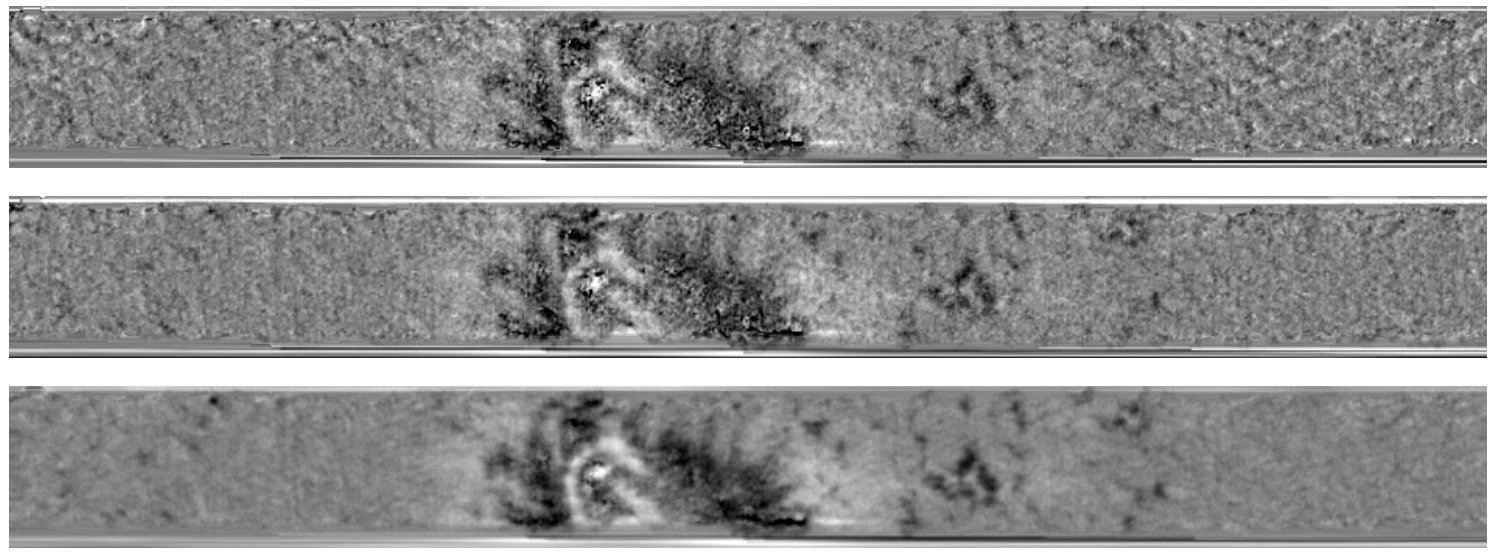

Fig. 8. Line of sight magnetic field $B / /$ in $\mathrm{NaD} 1 \pm 288 \mathrm{~m} \AA$. North polarities are white and south polarities are dark. Top: $B / /$ is derived directly from the difference of line shifts measured on $I+V$ and $I-V$; middle: $B / /$ is obtained by the same method, but after cross correlation and destretching; bottom: $B / /$ is computed from a burst of 10 couples $I+V, I-V$, taking the mean value of the data after cross correlation and destretching, hence reducing noise.

noticed a good spatial correlation between downflows and magnetic elements (see Fig. 9, right), although the $5 \mathrm{~min}$ oscillations have not been filtered (we do not have a temporal sequence). The intensity of the magnetic field measured in magnetic concentrations at $\pm 288 \mathrm{~m} \AA$ is twice the one obtained at $\pm 144 \mathrm{~m} \AA$, and it is also the case for velocities: this result suggests that the cross section of magnetic tubes has increased approximately by a factor 2 between the two heights of observation separated by roughly $160 \mathrm{~km}$. Such a result corroborates previous works, such as the one by Eibe et al. (2002) over an active region observed with the MSDP at Themis in the same spectral line, but at lower spatial resolution. Such a divergence with height is not in agreement, according to these authors, with potential or force free fields.

- In the second method, we do not superimpose data coming from the same pixel of $I+V$ and $I-V$ maps, but we try to correct at first the effects of atmospheric 

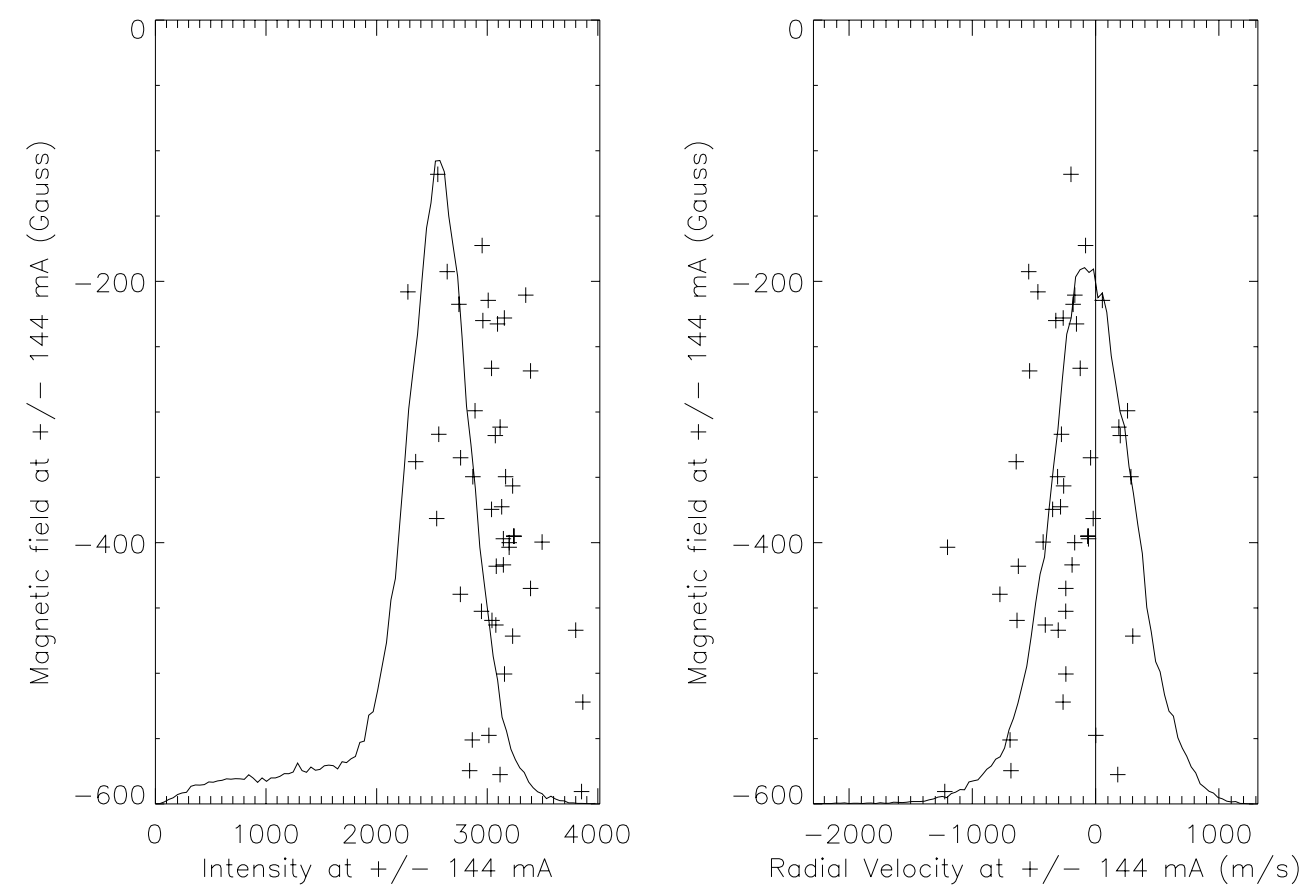

Fig. 9. The magnetic field measured at $\pm 144 \mathrm{~m} \AA$ as a function of intensity (left) and radial velocity (right); crosses represent the 47 magnetic elements selected outside the sunspot. For each picture, the histogram of intensity fluctuations and radial velocities of the full observed area of $16 \times 142$ arcsec is drawn, for comparison.

turbulence. The continuum images are cross correlated and destretched, and the result is then applied to the maps of line shifts derived from $I+V$ and $I-V$ data. As shown by Figs. 7 and 8 (middle), the noise is reduced, particularly in quiet regions, without affecting significantly the spatial resolution nor the strong fields measured in magnetic concentrations. This can be probably explained by the quality of the seeing conditions and the short time lag between subsequent frames $(0.25 \mathrm{~s})$, as the correction provided by our procedure does not exceed half a pixel.

- In the third method, we use the full burst ( 10 couples $I+V$, $I-V)$ in order to reduce the noise in the magnetic data. The first continuum image of the burst is taken as a reference frame, and subsequent continuum images are cross correlated and destretched in comparison with the first one. Then, the results are applied to line shift maps. Each pair of $I+V, I-V$ data provides a map of the magnetic field which can be now summed or averaged in order to decrease the noise by a factor roughly equal to $\sqrt{10}$. Of course, such a procedure would be more interesting with long bursts (100 pairs for instance), but we were unable to do that at the time of observation. As shown by Figs. 7 and 8 (bottom), the noise is strongly reduced everywhere, but spatial resolution is affected, decreasing the values of the magnetic field. This last result may be due to seeing fluctuations during the burst which are not perfectly corrected by our procedure. Hence, this method seems more convenient for measuring weak fields at moderate spatial resolution.

The spatial resolution we got was estimated from cross sections of magnetic elements such as the one presented in Fig. 10. This figure emphasizes one of the finest magnetic elements in the

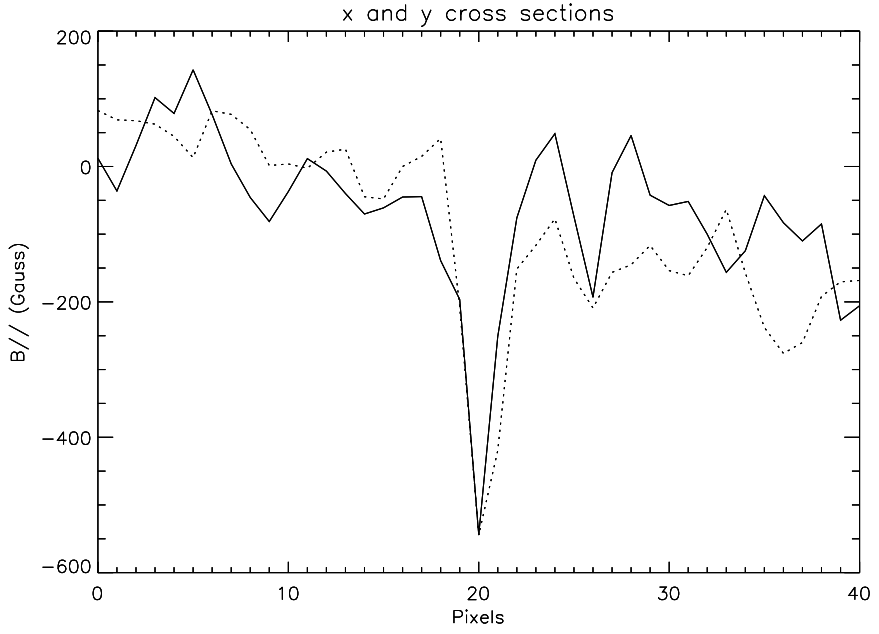

Fig. 10. Cross section in $x$ (solid line) and $y$ (dotted line) directions (parallel to the edges of the field of view) of the magnetic field $B / /$ in $\mathrm{NaD} 1 \pm 144 \mathrm{~m} \AA$. In abscissa, one tick mark represents the output pixel used in our data analysis $(0.15 \operatorname{arcsec})$.

vicinity of the sunspot and shows that the spatial resolution of our observation $(0.4 \operatorname{arcsec})$ is only limited by the pixel sampling, giving good hope to approach in the future the theoretical resolution of the refractor at this wavelength.

The noise of the data was estimated from the burst of $10 \mathrm{I}+$ $V, I-V$ pairs obtained in a few seconds, so representing several realizations of the same event. We consider for that purpose that the 10 maps of $B / /$ can be superimposed, after cross correlation and destretching by reference to the first frame of the burst. We computed the standard deviation $\sigma$ of the observed magnetic field $B / /$; it is on average over the full area of about 50 Gauss 


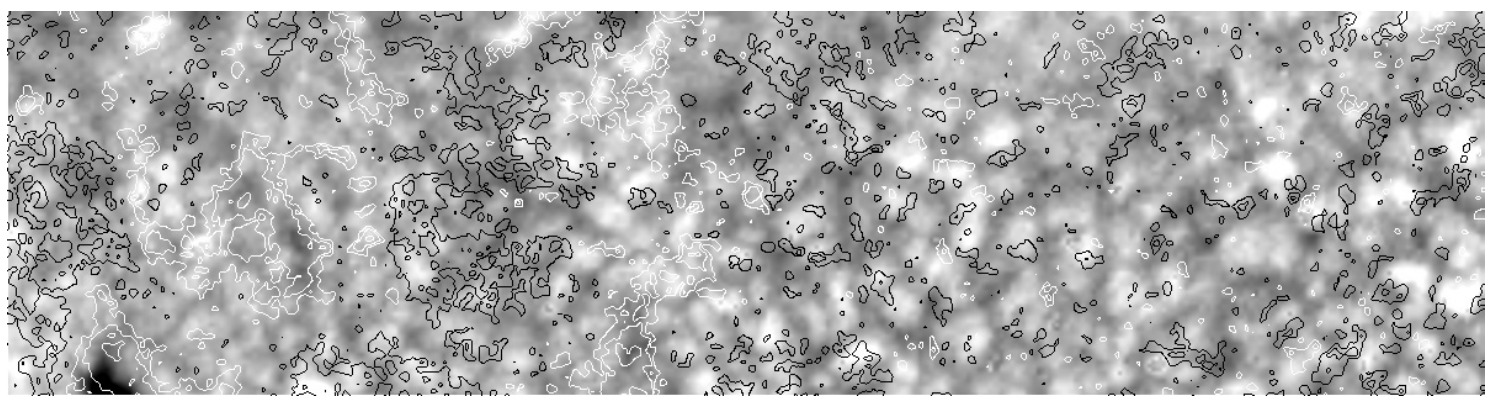

Fig. 11. Isocontours of the line of sight magnetic field (125 G, $250 \mathrm{G}, 500 \mathrm{G})$, negative values in black and positive values in white, superimposed to the continuum intensity (MSDP channel 11). The sunspot is outside the represented area (at left). The size of this reduced field of view is $16 \times 70$ arcsec.

at $\pm 144 \mathrm{~m} \AA$ and 130 Gauss at $\pm 288 \mathrm{~m} \AA$. This error bar includes all instrumental and seeing effects such as turbulence which were not corrected by destretching. This means that, if we take a confidence interval of $68 \%$ (respectively $95 \%$ ), the error on the average magnetic field derived from the burst of $10 I+$ $V, I-V$ couples is about $\sigma / \sqrt{10}$ (respectively $2 \sigma / \sqrt{10}$ ), i.e. numerically 16 Gauss (respectively 32 Gauss) at $\pm 144 \mathrm{~m} \AA$ (see Fig. 7, bottom).

We have plotted in Fig. 11 isocontours of the magnetic field superimposed on the continuum, only for the region located West of the sunspot, where the granulation pattern is clearly visible. The figure reveals that magnetic elements are preferentially located in intergranular lanes, which confirms previous results. But we did not find evidence of very good correlation between bright points (such as the filigree) visible in the line at $\pm 144 \mathrm{~m} \AA$ and magnetic elements (see Fig. 9, left), as already noticed in previous works (Zhang et al. 1998; Keller 1992). In particular, Keller (1992) found that magnetic features larger than 0.4 arcsec in size tend to be darker than their surroundings. Muller et al. (2000) and Zhang et al. (1998) found that the bright filigree is related to magnetic features, but it is not generally cospatial. However, Fig. 9 shows that magnetic concentrations are preferentially associated with downflows and intensities brighter than the median, when measured at the same band in the spectral line.

\section{Conclusion}

We have obtained observations of the line of sight magnetic field over a $2 \mathrm{D}$ field of view at high spatial resolution $(0.4$ arcsec $)$ with the new FLC polarimeter installed at the Pic du Midi Turret Dome. We focused our attention on the granular pattern in the vicinity of active region NOAA 0459. We found many magnetic concentrations, mainly located in the intergranular network. We measured magnetic fields of a few hundred Gauss, contrarily to Dominguez Cerdeña et al. (2003), but they were observing in the internetwork, far from active regions. The MSDP technique allowed us to compute magnetograms at two different levels of altitude. It appears clearly that the magnetic fields measured in the $\mathrm{NaD} 1$ wings are stronger than those obtained from line centre, suggesting that magnetic lines diverge with increasing height. We noticed that magnetic concentrations are not strictly superimposed at the two altitudes, suggesting that magnetic lines could be inclined or curved. We noticed also that magnetic concentrations are almost systematically associated with downflows in the range of a few hundred meters per second and often appear bright near the line centre only. On the contrary, we were not able to confirm any relationship between magnetic concentrations and bright points in the continuum, for the reason that we did not find, at the spatial resolution of 0.4 arcsec, clear evidence of such points. Higher resolution is probably required, as suggested by Roudier et al. (2003) showing evidence of numerous bright points in non polarimetric NaD1 MSDP observations, but with a smaller pixel than the one we have here.

During the 2004 campaign, we will perform new polarimetric observations of the granular pattern with a better spatial sampling ( 0.13 arcsec instead of 0.20 arcsec) in order to be able to approach the theoretical resolving power of the $50 \mathrm{~cm}$ refractor. Our objective is now to get a temporal sequence, with $45 \mathrm{~s}$ resolution, in order to study evolution of the dynamics and the magnetometry of solar granulation. With the new computer system installed in December 2003 at the Turret Dome, we now have the possibility to make bursts of 100 pairs of MSDP images $(I+V, I-V)$ at high observing cadence ( 8 images/s). This new facility will increase the efficiency of image selection and also will have an impact on magnetic sensitivity. For instance, our aim is to reach a sensitivity better than 10 Gauss from data analysis and destretching of bursts of 100 pairs obtained in less than $45 \mathrm{~s}$. We also plan to incorporate speckle restoration of long polarimetric bursts, following Dominguez Cerdeña et al. (2003).

We know that our first version polarimeter has many defaults and limitations which need to be corrected and improved in the future, such as chromatism and temperature variation, producing in particular moving fringes. Also, this focal device can measure only some components of the Stokes vector ( $I$ and $V$ with the static quarter wave plate, $I$ and $Q$ without it). For this reason, we will develop a second version of our FLC polarimeter, including two modulators, which will be able to sequentially measure the full Stokes vector $(I, Q, U, V)$. We will also increase the accuracy of measurements with the use of temperature controlled variable retarders, the retardation being exactly adjusted to the wavelength of the observed spectral line.

The spectral resolution of observations will be improved in the future. In the MSDP technique, it is limited by the 
resolution of the multi slit beam shifter located on the spectrum. With a new optical design, we will reduce the wavelength shift between consecutive channels to only $60 \mathrm{~m} \AA$, allowing observations of various chromospheric or photospheric lines, such as Fe I $630.1 \mathrm{~nm}$ or Ca I $610.3 \mathrm{~nm}$.

\section{Additional information}

A Power Point file, presenting with better quality some of the figures of this paper, together with additional animations, is available at the following URL:

http://helios.obspm.fr/malherbe/

AA20030919/AA20030919.ppt

Acknowledgements. We want to express our gratitude to Ch. Coutard, engineer in optics, for his invaluable help in the optical setup of the polarimeter and the MSDP spectrograph, and his contribution to observations. We are also indebted to M. Lafon and F. Grimaud for their help in data processing. The spectrograph was designed by Dr. Z. Mouradian under the auspices of Observatoire de Paris and INSU/CNRS. We wish also to thank the anonymous referee for helpful comments and suggestions that improved the manuscript.

This work was supported by Observatoire de Paris, Observatoire Midi Pyrénées, CNRS (UMR 5572 and UMR 8109) and the Programme National Soleil Terre (PNST). Special thanks are due to the Pic du Midi Observatory staff for their technical assistance during the observing run.

\section{References}

Berger, T. E., \& Title, A. M. 2001, ApJ, 553, 449

Cattaneo, F. 1999, ApJ, 515, L39
Cattaneo, F., Lenz, D., \& Weiss, N. 1999, ApJ, 563, 91

De Pontieu, B. 2002, ApJ, 569, 474

Dominguez Cerdeña, I., Sanchez Almeida, J., \& Kneer, F. 2003, A\&A, 417,741

Dominguez Cerdeña, I., Kneer, F., \& Sanchez Almeida, J. 2003, ApJ, 582, L55

Eibe, M. T., Aulanier, G., Faurobert, M., Mein, P., \& Malherbe, J. M. 2002, A\&A, 381, 290

Emonet, Th., \& Cattaneo, F. 2001, ApJ, 560, L197

Fligge, M., \& Solanki, S. 2000, JApA, 21, 275

Keller, C. U. 1992, Nature, 359, 307

Koschinsky, M., Kneer, F., \& Hirzberger, J. 2001, A\&A, 365, 588

Mein, P. 1981, Proc. Japan France seminar on solar physics, ed. Henoux \& Moriyama, 285

Mein, P. 1991, A\&A, 248, 669

Mein, P. 2002, A\&A, 381, 271

Muller, R., Dollfus, A., Montagne, M., Moity, J., \& Vigneau, J. 2000, A\&A, 359, 373

Muller, R., \& Roudier, Th. 1984, Sol. phys., 94, 33

Nisenson, P., van Ballegooijen, A. A., de Wijn, A. G., \& Sütterlin, P. 2003, ApJ, 587, 458

November, J. L. 1986, Appl. Opt., 25(3), 392

Roudier, Th., Eibe, M.-T., Malherbe, J.-M., et al. 2001, A\&A, 368, 652

Roudier, Th., Malherbe, J.-M., Mein, P., et al. 2003, A\&A, 409, 793

Schrijver, C. J., \& Zwann, C. 2000, Irish Astron. J., 27(2), 234

Steiner, O., Grossman-Doerth, U., Knoelker, M., \& Schüssler, M. 1998, ApJ, 495, 468S

Stolpe, F., \& Kneer, F. 2000, A\&A, 353, 1094S

Title, A. M., \& Berger, T. E. 1998, ApJ, 463, 797

Topka, K. P., Tarbell, T. D., \& Title, A. M. 1992, ApJ, 396, 351

van Ballegooijen, A. A., Nisenson, P., Noyes, R. W., et al. 1998, ApJ, 509,435

Zhang, H., Scharmer, G., Lofdahl, M., \& Yi, Z. 1998, Sol. Phys., 183, 283 